\title{
Giant retroperitoneal liposarcoma during pregnancy: case report
}

\author{
Dhruba Prasad Paul, Kashish Garg*
}

Department of Obstetrics and Gynecology, Agartala Government Medical College and GBP Hospital, Agartala, Tripura, India

Received: 22 September 2018

Accepted: 06 October 2018

\section{*Correspondence:}

Dr. Kashish Garg,

E-mail: kashish201992@gmail.com

Copyright: $($ ) the author(s), publisher and licensee Medip Academy. This is an open-access article distributed under the terms of the Creative Commons Attribution Non-Commercial License, which permits unrestricted non-commercial use, distribution, and reproduction in any medium, provided the original work is properly cited.

\begin{abstract}
24-year-old woman was presented with huge vulval mass for which she was operated, histopathological report of that mass shows angiomyoma. After few months she was discovered to have a giant retroperitoneal tumor incidentally during her routine obstetric examination at 24 weeks of gestation. Initial investigation by abdominal-pelvic computed tomography (CT) (18/08/18) revealed an a large multiseptated soft tissue attenuated minimally enhancing pelvic mass $(31.8 \mathrm{~cm} \times 13.2 \times 24 \mathrm{~cm})$ protruding in the abdominal cavity displacing the bowel loops proximally and pushing retro organs posteriorly. She underwent laparotomy with preservation of the fetus at 24 weeks of gestation. Final diagnosis was made after HPE report which shows myxoid liposarcoma. She was referred to regional cancer hospital for radiotherapy and then patient lost in follow up.
\end{abstract}

Keywords: Angiomyoma, HPE, Myxoid liposarcoma, Radiotherapy, Retroperitoneal tumor, Vulval mass

\section{INTRODUCTION}

In some cases, pregnancy can occur with large tumor masses in the abdomen. This compromises the pregnancy and asks for premature delivery or abortion, depending on when the tumor mass is diagnosed. The abdominal tumors which occur during pregnancy may be of gynecologic origin, other abdominal tumors, lymphomas or other undiagnosed abdominal masses. These masses need to be operated as soon as the pregnancy ends. Liposarcoma in pregnancy are peculiar clinical scenarios that are continuously being reported in the English literature for their rarity, challenges in management and speculations of association between pregnancy and disease kinetics. The World Health Organization classified liposarcomas into well-differentiated, myxoid, round-cell, pleomorphic and dedifferentiated subtypes; the first two subtypes belong to the low-grade lesions with better prognosis and higher incidence rates, which largely depends on the histologic subtypes, resection margin and age of the patients. ${ }^{1,2}$ With free surgical margin, the 5-year overall survival rate can be $68-80 \%$, although the local recurrence rate $75 \% .2,3$ The peak prevalence of retroperitoneal liposarcoma (RPLS) is between the age of 40 to 70 years, usually seen in male 1.43:1. Liposarcoma in pregnancy is rare but deserves practitioners' attention, for early detection and proper surgical management could greatly improve disease outcomes.

\section{CASE REPORT}

A 24 years old women Mrs. X, residing at Ataharakant, Shantinagar Radhapur attended our hospital in the gynae OPD with complain of rapidly enlargement of vulva for last 6-7 months along with difficulty in passing stools and urine. After examination and discussion decision was made and patient was referred to surgery department. She was operated for the same huge vulval mass around $15 \times 10 \mathrm{~cm}$ in the month of March 2017. 
Post op uneventful. Patient was discharged normally and advised to follow up monthly. Later on, biopsy report of that mass shows features suggestive of angiomyoma. In December 2017 she had again come with distention of abdomen for last 4 months with difficulty in breathing, walking, sleeping along with 5months of amenorrhoea. She had history of 2 miscarriages, the cause of the recurrent miscarriage not known. Patient had menarche at the age of 14, after she had regular periods. Besides that, her past medical history was unremarkable. She did not remember her LMP exactly. During a routine obstetric examination, separate uterus not palpable as abdomen was hugely distended. Her body mass index (BMI) was 21.99 before this pregnancy when she presented to our hospital at 23 weeks pregnancy (by early week scan) her BMI was 30.09 with a good nutritional status. USG Shows (18/08/17)- A gestation sac around 6weeks 6days size seen with in endometrial cavity, yolk sac seen with in gestational sac, large multi-septed solid SOL with few hyperechoic and cystic lesion seen in peritoneal cavity.

The patient was then submitted to abdominal-pelvic computed tomography (CT) (18/08/17) revealed an a large multiseptated soft tissue attenuated minimally enhancing pelvic mass $(31.8 \mathrm{~cm} \times 13.2 \times 24 \mathrm{~cm})$ protruding in the abdominal cavity displacing the bowel loops proximally and pushing retro-organs posteriorly. However, there was no evidence of direct invasion to adjacent organ or metastasis. Laboratory findings showed mild leukocytosis and mild anaemia and other laboratory analysis revealed normal values of hepatic-renal function, urinalysis and electrolytes. Tumor marker studies showed increased level of CA-125 of $105.40 \mathrm{U} / \mathrm{mL}$ (normal range, 0 to 35 ), $3-\mathrm{HCG}-23,388 \mathrm{IU} / \mathrm{ml}$. At the time of diagnosis, the size of tumor was very large $(31 \times 13 \times 24$ $\mathrm{cm})$ and it was rapidly enlarging with high chance of being malignant tumor. It was determined that an early removal of the tumor will enhance the safety of the patient as well as the fetus. After a thorough discussion it was decided to perform the tumor removal along with continuation of pregnancy.

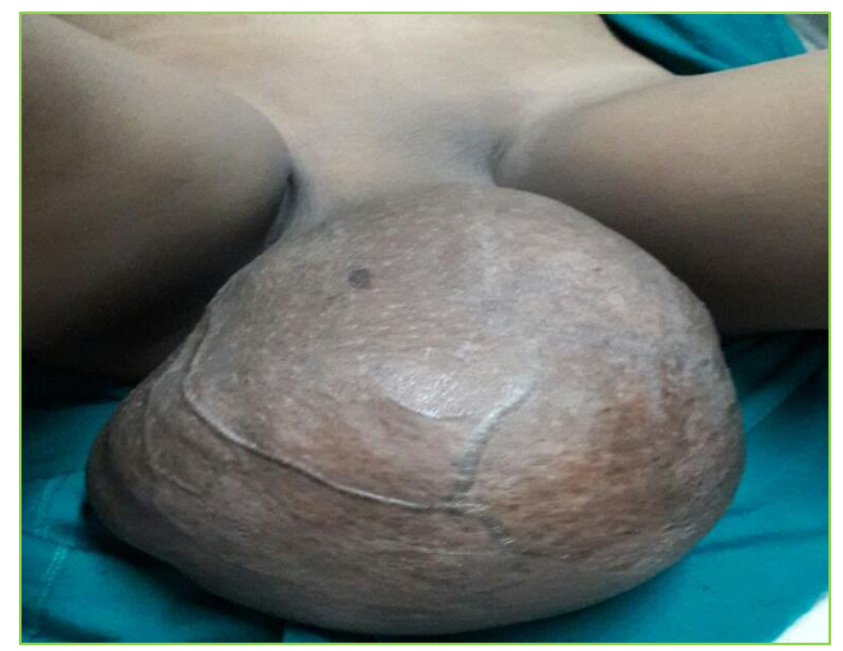

Figure 1: Huge vulval mass.

\section{Intra-op findings}

Abdomen opened through infra-umbilical vertical incision which was extended beyond umbilicus to assess the whole lump. Pregnant uterus was retracted anteriorly (24-26weeks size). Both sides tubes and ovaries normal. Jelly like masses was found intraperitoneally which appeared to arise from intra-peritoneum around $15 \mathrm{kgs}$ and masses sent for HPE. Hemostasis achieved and abdomen closed in layers. The patient had an uneventful recovery. The patient was discharged 15 days after surgical treatment without any immediate postoperative complication.

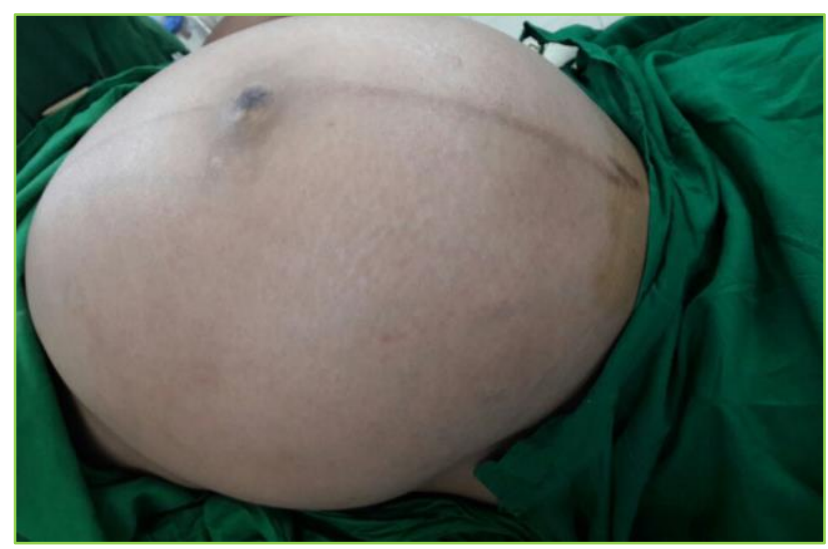

Figure 2: Abdomen before operation.

Later histopathological examination repost shows Myxoid liposarcoma. The patient presented with pain abdomen after 1 month of operation followed by spontaneous expulsion of fetus. After that she had no problem, but the patient was referred to regional cancer hospital for radiotherapy and then patient lost in follow up.

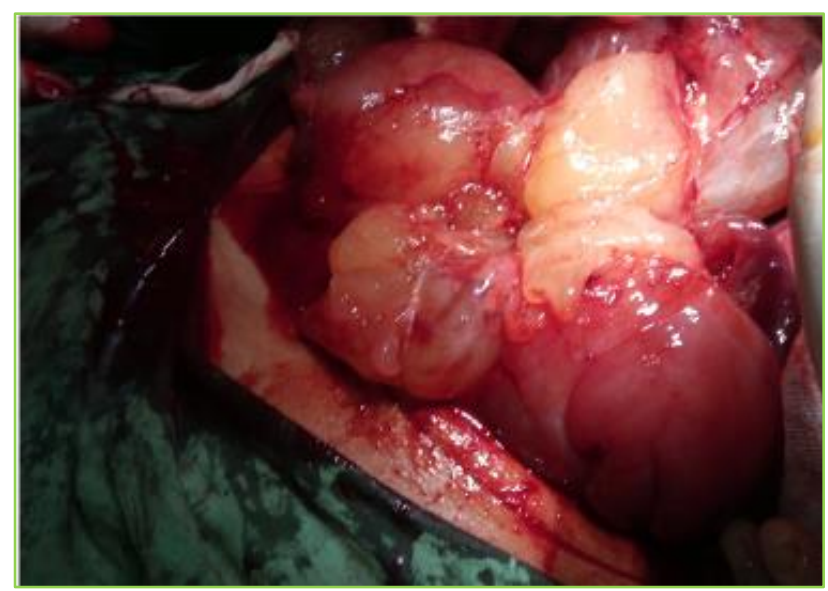

Figure 3: Intra-op findings.

\section{DISCUSSION}

RPLS is one of the most common types of soft tissue sarcoma with peak prevalence between the age of 40 to 
70 years, which is usually seen in male $1.43: 1 .^{4,5}$ RPLS during pregnancy is extremely rare and a challenge for both the surgeon and pregnant woman. ${ }^{6}$

Liposarcoma is categorized into five histological subtypes: well-differentiated, myxoid, round-cell, pleomorphic, and dedifferentiated (WHO classification, 2002). Well-differentiated and myxoid subtypes belong to the low-grade lesions, whereas the other subtypes are considered as high-grade lesions.

Myxoid liposarcoma is the second most common subtype, consists of $18 \%$ of all liposarcoma, and is the most common subtype in young patients. ${ }^{7}$ It is often found with painless palpable mass arising in the thigh and retroperitoneal space, especially for young patients. ${ }^{4} \mathrm{MRI}$ is a highly reliable radiological method in diagnosing these tumors, especially for assessment of pregnant woman. With careful interpretation, the MRI can provide us with enough information for accurate diagnosis differentiation and even identification of subtypes. ${ }^{8-10}$ Myxoid liposarcoma has a relatively characteristic appearance as a well-circumscribed multinodular mass with low signal intensity on $\mathrm{T}_{1} \mathrm{WI}$ and a high but heterogeneous intensity on $\mathrm{T}_{2} \mathrm{WI}$.

The relationship between pregnancy and liposarcoma is not clear. Cantin and Mc Neer considered that pregnancy does not adversely affect the prognosis of the tumor. Whereas, they suggested that an estrogen-progesterone environment possibly has a favorable impact on the natural history of sarcoma, and hormone therapy in the management of metastatic sarcoma should be explored. ${ }^{11}$

The retroperitoneal space is rather vast; RPLS has no specific symptoms in the early stage. Although surgery is the mainstay of the therapeutic modalities, the RPLS is usually diagnosed in the advanced stage with a palpable mass, and contiguous organ resection is often indicated. ${ }^{12}$ If resected with negative surgical margin, the 5-year overall survival rate can be $68 \%$ to $80 \%$, although local recurrence rate can be as high as $75 \%{ }^{12}$. The extended surgery, contiguous organ resection, major vessel resection, and reconstruction are often indicated to get a negative surgical margin and a better prognosis. ${ }^{13}$

This patient was diagnosed with giant retroperitoneal tumor incidentally during her routine obstetric examination. The therapeutic protocol is challenging because: 1) The patient has a firm willing of preserving the fetus. 2) The resection is urgent as the tumor is expanding. 3) The impact on the fetus should be assessed such as time of gestation, hemorrhage, length of operation, medications and anesthesia.

Authors reviewed the literature; only 16 cases of liposarcoma during pregnancy were reported in English literature, ten were derived from retroperitoneal space, and five cases are myxoid subtype. Surgery was performed in five cases after delivery, five cases concurrent with cesarean section. Three patients died within the first year after surgery. ${ }^{14-17}$ In only two cases, surgeries were performed during pregnancy in 13 weeks of gestation. ${ }^{18,19}$ Most surgery were performed postpartum or at the time of cesarean section.

\section{CONCLUSION}

In rare cases, pregnancy can be compromised by large intra-abdominal tumors which can put the health of both the mother and the fetus in danger. It's suggested that future mothers are regularly examined, prior to pregnancy, so that they can keep track of their health and ability to reproduce. The entire pregnancy period should be carefully observed, too, since the symptoms can sometimes lead to interdisciplinary examinations and consultations with other specialists. This will ensure mothers are healthy and can give birth to healthy offspring.

\section{ACKNOWLEDGMENTS}

Author would like to thank Professor Anup Saha, Head, Department of Surgery, Agartala Government Medical College for his help during surgery.

\section{Funding: No funding sources Conflict of interest: None declared \\ Ethical approval: Not required}

\section{REFERENCES}

1. Huo D, Liu L, Tang Y. Giant retroperitoneal liposarcoma during pregnancy: a case report. World J Surg Oncol. 2015;13:145.

2. Lopes RI, Machado M, Paz C, Santos AC, Rezende WW. Successful outcome of a surgically treated giant retroperitoneal liposarcoma during pregnancy. Arch Gynecol Obstet. 2009;280:1067-9.

3. Tebes S, Cardosi R, Hoffan M. Liposarcoma complicating pregnancy. Gynecol Oncol. 2001;83:610-2.

4. Enzinger CJ, Weiss SW. Soft tissue tumors. 3. St Louis: Mosby; 1995.

5. Dalal KM, Kattan MW, Antonescu CR, Brennan MF, Singer S. Subtype specific prognostic nomogram for patients with primary liposarcoma of the retroperitoneum, extremity, or trunk. Ann Surg. 2006;244(3):381-91.

6. Yamamoto $\mathrm{T}$, Marui $\mathrm{T}$, Akisue $\mathrm{T}$, Hitora $\mathrm{T}$, Kawamoto T, Nagira $\mathrm{K}$, et al. Management of liposarcoma occurring in pregnant women. Anticancer Res. 2003;23(1B):799-802.

7. Alaggio R, Coffin CM, Weiss SW, Bridge JA, Issakov J, Oliveira AM, et al. Liposarcomas in young patients: a study of 82 cases occurring in patients younger than 22 years of age. Am J Surg Pathol. 2009;33(5):645-58.

8. Jelinek JS, Kransdorf MJ, Shmookler BM, Aboulafia AJ, Malawer MM. Liposarcoma of the extremities: 
MR and CT findings in the histologic subtypes. Radiol. 1993;186(2):455-9.

9. Sung MS, Kang HS, Suh JS, Lee JH, Park JM, Kim JY, et al. Myxoid liposarcoma: appearance at MR imaging with histologic correlation. Radiographics. 2000;20(4):1007-19.

10. Murphey MD, Arcara LK, Fanburg-Smith J. From the archives of the AFIP: imaging of musculoskeletal liposarcoma with radiologic-pathologic correlation. Radiographics. 2005;25(5):1371-95.

11. Cantin J, McNeer GP. The effect of pregnancy on the clinical course of sarcoma of the soft somatic tissues. Surg Gynecol Obstet. 1967;125(1):28-32.

12. Strauss DC, Hayes AJ, Thway K, Moskovic EC, Fisher C, Thomas JM. Surgical management of primary retroperitoneal sarcoma. $\mathrm{Br} \mathrm{J}$ Surg. 2010;97(5):698-706.

13. Fiore M, Colombo C, Locati P, Berselli M, Radaelli $\mathrm{S}$, Morosi C, et al. Surgical technique, morbidity, and outcome of primary retroperitoneal sarcoma involving inferior vena cava. Ann Surg Oncol. 2012;19(2):511-8.

14. Foruhan B. Retroperitoneal sarcomas obstructing delivery: two case reports. $\mathrm{Br} \mathrm{J}$ Obstet Gynaecol. 1979;86(9):747-8.

15. De Jaco P, Giorgio M, Zantedeschi B, Mazzoleni G, Marabini A. A case of retroperitoneal liposarcoma in pregnancy. Acta Obstet Gynecol Scand. 1993;72(2):122-4.

16. Matsuda S, Tanaka K, Harimaya K, Matsumoto Y, Sato H, Iwamoto Y. Treatment of myxoid liposarcoma in pregnancy. Clin Orthop Relat Res. 2000;376:195-9.

17. Tebes S, Cardosi R, Hoffman M. Liposarcoma complicating pregnancy. Gynecol Oncol. 2001;83(3):610-2.

18. Lopes RI, Machado M, Paz C, Santos AC, Rezende WW. Successful outcome of a surgically treated giant retroperitoneal liposarcoma during pregnancy. Arch Gynecol Obstet. 2009;280(6):1067-9.

19. Kurogouchi A, Hyodo MSH, Yamaguchi T, Niki T, Nagai H. Long-term survivor following resection of a retroperitoneal myxoid liposarcoma during pregnancy (in Japanese with English abstract) Nihon Rinsyogeka Gakkaizasshi. Jpn J Surg Assoc. 2011;72:2129-33.

Cite this article as: Paul DP, Garg K. Giant retroperitoneal liposarcoma during pregnancy: case report. Int J Reprod Contracept Obstet Gynecol 2018;7:4768-71. 